\title{
Bases para expansão e desenvolvimento adequados de programas de Residência em Medicina de Família e Comunidade
}

\author{
Bases for an adequate expansion and \\ development of Family and Community \\ Medicine Residency Programs
}

\author{
Maria Inez Padula Anderson* \\ Eno Dias de Castro Filho** \\ Ricardo Donato Rodrigues*** \\ Marcello Dala Bernardina Dalla**** \\ Monique Marie Marthe Bourget*****
}

\section{Resumo}

Este artigo é uma versão adaptada, para este número suplementar da Revista Brasileira de Medicina de Família e Comunidade, do documento original da Sociedade Brasileira de Medicina de Família e Comunidade (SBMFC), editado em 2005, sob o mesmo título - disponível na íntegra no portal wmw.sbmfc.org.br. O texto situa a Medicina de Família e Comunidade (MFC) como uma especialidade médica centrada no paradigma da integralidade biopsicossocial que prioriza a APS, fazendo um nítido contraste com as especialidades focais. As políticas de saúde historicamente adotadas no Brasil privilegiaram, no entanto, um modelo assistencial hospitalocêntrico, bloqueando o desenvolvimento da APS e da MFC. Esta é uma tendência em mudança em face da Estratégia Saúde da Família e da experiência internacional nesta área. Neste contexto, a SBMFC, em parceria com o MS, vem incentivando a expansão dos programas de residência em MFC. Considerando a importância de que tal expansão seja implementada sem prejuízo da qualidade, a SBMFC apresentou um conjunto de critérios e recomendações, sintonizadas com a Resolução da CNRM - que regulamenta estes programas -, para que servissem de base para tal expansão. Neste artigo, são retomadas as exigências e recomendações relativas à organização do programa, à distribuição temporal das atividades e às competências básicas que deverão ser desenvolvidas ao longo do programa de formação. Além disso, são revistos os requisitos referentes à infra-estrutura e ao fluxo assistencial necessários para criar um ambiente favorável de treinamento em serviço. Por último, faz-se um pequeno relato sobre as iniciativas mais recentes que a SBMFC vem desenvolvendo no sentido de minorar a atual defasagem entre necessidade e oferta de preceptores especializados em MFC.

Palavras-chave: Ação Intersetorial; Saúde da Família; Política de Saúde.
Key Words: Intersectorial Action; Family Health;

Health Policy.

*Médica de Família e Comunidade, Doutora em Saúde Coletiva, Professora adjunta e preceptora da Residência em MFC, FCM, UERJ, Presidente da SBMFC, Rio de Janeiro, Brasil.

**Médico de Família e Comunidade, Mestre em Educação, Diretor Científico SBMFC 2004-2008, Coordenador Telessaúde RS.

***Médico, Doutor em Medicina. Prof. Adj. Programa de Atenção Primária em Saúde da FM UFRJ.

****Médico de Familia e Comunidade. Mestre em Educação. Prof da Escola Superior de Ciências da Santa Casa de Misericórdia de Vitória ES e do Centro Universitário de Vila Velha. Vice presidente da SBMFC.

*****Médica de Família e Comunidade. Coordenadora do PSF Santa Marcelina, SP. Vice Presidente da APMFC. 
Maria Inez Padula Anderson, Eno Dias de Castro Filho, Ricardo Donato Rodrigues, Marcello Dala Bernardina Dalla e Monique Marie Marthe Bourget
Bases para expansão e desenvolvimento adequados de programas de Residência em Medicina de Família e Comunidade

\begin{abstract}
This article is a version of the original document published in 2005 under the same title by the Brazilian Society of Family and Community Medicine (BSFCM) (full text available at <wmw.sbmfc.org.br>), adapted for this supplement of the Revista Brasileira de Medicina de Familia e Comunidade. The text defines Family and Community Medicine (FCM) as a medical specialty focused on biopsychosocial integrality an on Primary Care as privileged scenario, in distinct opposition to the medical specialties. The health policies historically adopted in Brazil used to give priority to a hospital-centered care model, hampering the strengthening of Primary Care and Family and Community Medicine. This trend however is changing due to the implementation of the Family Health Strategy and the international experience in this area. In this context, the BSFCM in cooperation with the Ministry of Health are encouraging the expansion of the Family and Community Medicine Residency Programs. Considering the importance of expanding the residency programs without loosing quality, the BSFCM elaborated a document presenting a set of criteria and recommendations in accordance with the guidelines of the National Commission of Medical Residency, the organism regulating these programs, to be used as a basis. In this article we recover the requirements and recommendations for the organization of the program, the chronogram of activities and the basic competences to be developed during the program. Furthermore we reanalyze the requisites in terms of infrastructure and service flow necessary for creating a practice environment favorable for in-service training. We finalize with a brief report about the most recent actions of the BSFCM for minimizing the current gap between offer and demand of preceptors specialized in Family and Community Medicine.
\end{abstract}

\section{Introdução.}

Este artigo é uma versão adaptada e atualizada do documento original da Sociedade Brasileira de Medicina de Família e Comunidade (SBMFC), editado em 2005, sob o mesmo título - disponível na íntegra no portal wmw.sbmfc.org.br.

Aquele documento define Medicina de Família e Comunidade (MFC) como uma especialidade médica da integralidade, cujo cenário privilegiado de prática é a Atenção
Primária à Saúde (APS). Esta especialidade está voltada para o cuidado em saúde às pessoas, famílias e comunidades de forma abrangente, continuada e contextualizada, preferencialmente no âmbito de uma equipe multidisciplinar. $\mathrm{O}$ médico de Família e Comunidade deve estar habilitado para cuidar com resolutividade dos problemas de saúde mais freqüentes da população a ele referenciada, sem distinção de idade ou gênero.

Como especialidade médica presente em grande número de países, especialmente entre as nações mais ricas do hemisfério norte, a MFC tem contribuído para a implantação de mudanças nos sistemas de saúde com vistas à inclusão de todos com melhoria de qualidade. Em nosso país, desde seu reconhecimento como especialidade médica, em 1981, a MFC está comprometida com o conceito de excelência no âmbito da APS, inclusive da clínica, e com os preceitos da Reforma Sanitária brasileira.

Insere-se, portanto, no esforço da sociedade brasileira visando à consolidação do Sistema Único da Saúde (SUS), enquanto sistema público, democrático e universal, constituído por uma rede de serviços no qual a APS seja capaz de propiciar fácil acesso a todos, dispensar cuidados abrangentes ao longo de toda a vida e ordenar o fluxo de pacientes pelos serviços dos diferentes níveis de atenção, na medida do necessário.

A reforma do setor saúde brasileiro teve um grande impulso quando o Ministério da Saúde decidiu priorizar a Atenção Primária à Saúde como base de estruturação do SUS por meio da Estratégia da Saúde da Família.

Entretanto, antes deste processo mais recente, entre outros paradoxos das políticas de saúde adotadas no país, havia ocorrido, no final da década de 1970, no curso da Reforma Sanitária, um retrocesso no movimento de expansão dos Programas de Residência de Medicina de Família e Comunidade (então denominada Medicina Geral Comunitária).

Naquela época, a escala de formação de especialistas no campo da Atenção Primária à Saúde, que se vinha expandindo, foi severamente reduzida pelo corte de financiamento de grande parte dos Programas de Residência 
em Medicina Geral Comunitária, pelo então Instituto de Previdência Social ${ }^{1}$.

Os efeitos negativos dessa medida tornaram-se mais visíveis com a expansão acelerada da Estratégia Saúde da Família no país. Médicos especialistas focais voltados para o atendimento de problemas de saúde que afetam órgãos e sistemas, gêneros e faixas etárias acabaram assumindo maciçamente tarefas no âmbito do cuidado integral sem, contudo, estarem preparados para $\mathrm{tal}^{2,3,4}$, e isso quase sempre ocasiona importante carga de sofrimento para os próprios médicos, para as equipes e população.

Em face da necessidade de qualificação e expansão da Estratégia Saúde da Família, a SBMFC e o Ministério da Saúde vêm estabelecendo parcerias, debatendo e avaliando estratégias que articulem maior oferta de programas de formação de especialistas, trabalho em equipe e expansão do acesso da população ao Saúde da Família.

Entre as alternativas pactuadas vale destacar o programa de incentivo do Ministério da Saúde à implantação de novos PRMFC, especialmente àqueles em Unidades Básicas de Saúde em parceria com as Secretarias Municipais de Saúde, que previa a complementação do valor das respectivas bolsas pelo MS, aprovado no Congresso Nacio$\mathrm{nal}^{5,6,7,8,9}$.

Com essa e outras estratégias, esperava-se uma reversão progressiva daquele paradoxo, antes mencionado, através da rápida multiplicação do número de programas.

Visando a contribuir para o aperfeiçoamento e a expansão desta formação, a SBMFC empenhou-se na organização deste documento, a partir de sua especificidade como sociedade científica e sua co-responsabilidade institucional quanto à Residência Médica, definida no âmbito da CNRM.

Vale notar que o processo de implantação de novos PRM, em situações e modus operandi diferenciados predominantemente fora do ambiente hospitalar -, torna necessário um acompanhamento ainda mais cuidadoso deste processo. Sugere ainda a necessidade de uma particularização de normas complementares e transitórias até que o desenvolvimento dos programas adquira maturidade sufici- ente.

Vencida esta primeira etapa, ainda restarão, como para qualquer outro programa, os desafios permanentes com vista à qualidade da formação oferecida, sua avaliação e melhoria contínua.

\section{Como formar um MFC por meio de Programas de Residência Médica? - Princípios}

A SBMFC entende que o especialista em MFC não é adequadamente formado com uma fusão antipedagógica das chamadas áreas básicas, como pediatria, ginecoobstetrícia, medicina interna, cirurgia e psiquiatria. Ao contrário, esta formação deve inspirar-se em um modelo de prática médica centrada na pessoa e na relação médicopaciente, com foco na família e orientada para a comunidade, privilegiando, por conseqüência, o primeiro contato, o vínculo, a continuidade e a integralidade do cuidado na atenção à saúde ${ }^{10}$.

O egresso de um programa de residência, organizado em sintonia com a concepção acima, deverá ser capaz de desenvolver ações integradas de promoção, proteção e recuperação da saúde, tanto no nível individual, quanto familiar e comunitário. Para tanto, é necessário o desenvolvimento de conhecimentos e habilidades técnicas específicas, de modo que a formação do MFC deve ocorrer com preceptores especializados em MFC, mais experientes e pedagogicamente habilitados para o ensino-aprendizado com adultos jovens.

Com o objetivo de colaborar para que estes novos e, também, os antigos programas de RMFC se desenvolvam com base em uma estrutura programática e didáticopedagógica coerente e qualificada, a Diretoria da SBMFC elaborou o presente documento.

Neste, encontram-se elementos considerados necessários ao processo de estruturação de programas de residência em Medicina de Família e Comunidade, incluindo as competências básicas que deverão ser constituídas ao longo do programa de formação e a distribuição temporal das atividades.

Além disso, assinala os elementos da infra-estrutura 
e de fluxo assistencial, necessários para criar um ambiente favorável de treinamento em serviço. $\mathrm{Na}$ seqüência, propõe um conjunto de iniciativas que vem desenvolvendo no sentido de minorar a atual defasagem entre necessidade e oferta de preceptores especializados em MFC.

\section{Organização e desenvolvimento dos Programas de Residência Médica em MFC}

Recomendações para a distribuição de carga horária de acordo com as atividades práticas e teóricas a serem desenvolvidas.

De acordo com as normas da Comissão Nacional de Residência Médica (CNRM) os programas de residência se desenvolvem ao longo de, no mínimo dois anos, com carga horária semanal de $60 \mathrm{~h}^{11}$.

Considerando as 52 semanas, e o período regulamentar de férias do residente (quatro semanas/ano), a carga horária total do treinamento em serviço é de $2.880 \mathrm{~h} /$ ano (60h X 48 semanas) ou de 5.760h/dois anos.

Para facilitar o cálculo dos períodos de tempo a serem atribuídos às atividades, deve ser considerada a carga horária anual total e distribuí-la por meio do período, na forma de atividades contínuas e/ou concentradas em períodos, de acordo com o tipo de atividade e sua pertinência.

Para facilitar a distribuição da carga horária, o percentual atinente a cada atividade deve ser planejado em percentuais, divisíveis ou múltiplos de 5 (1, 25\%, 2,5\%, $5 \%$, $10 \%)$, considerando a carga horária anual total (2.880h). Uma vez calculada, pode-se distribuir por semana, mês, semestre ou ano, de acordo com o mais adequado em termos de treinamento.

Por exemplo: uma atividade que requeira $40 \%$ da carga horária de $2.880 \mathrm{~h}$, ocupará $1.152 \mathrm{~h} /$ ano a serem distribuídas em 48 semanas, que podem corresponder a 24h/semana (seis turnos de 4 horas), 96 h/mês ou $576 \mathrm{~h} /$ semestre.

Quandro 1. Discriminação da carga horária das atividades práticas e teóricas dos PRMFC recomendada pela SBMFC, tendo em vista as resoluções da CNRM:

\begin{tabular}{|c|c|c|c|}
\hline Atividades & $\begin{array}{l}\text { Distribuição \% mínima } \\
\text { das } 2.880 \mathrm{~h} / \text { anuais em } \\
48 \text { semanas }\end{array}$ & $\begin{array}{l}\text { Distribuição \% máxima } \\
\text { das } 2.880 \mathrm{~h} / \text { anuais em } \\
48 \text { semanas }\end{array}$ & Totais \\
\hline Consultório de MFC & $\begin{array}{l}>/=40 \% \\
24 \mathrm{~h} / \mathrm{sem} . \text { ou } 1.152 \mathrm{~h} / \mathrm{ano}\end{array}$ & $\begin{array}{l}</=50 \% \\
28 \mathrm{~h} / \mathrm{sem} . \text { ou } 1.440 \mathrm{~h} / \text { ano }\end{array}$ & $\begin{array}{l}\text { Clínica da MFC } \\
50 \text { a } 65 \%\end{array}$ \\
\hline Atenção domiciliar & $\begin{array}{l}>/=5 \% \\
3 \mathrm{~h} / \mathrm{sem} . \text { ou } 144 \mathrm{~h} / \text { ano }\end{array}$ & $\begin{array}{l}</=15 \% \\
9 \mathrm{~h} / \mathrm{sem} . \text { ou } 432 \mathrm{~h} / \text { ano }\end{array}$ & \\
\hline Grupos terapêuticos & $\begin{array}{l}>/=5 \% \\
3 \mathrm{~h} / \mathrm{sem} . \text { ou } 144 \mathrm{~h} / \mathrm{ano}\end{array}$ & & \\
\hline $\begin{array}{l}\text { Administrativo- } \\
\text { gerenciais }\end{array}$ & $\begin{array}{l}>/=3,75 \% \\
2 \mathrm{~h} / \mathrm{sem} . \text { ou } 108 \mathrm{~h} / \mathrm{ano}\end{array}$ & $\begin{array}{l}</=10 \% \\
6 \mathrm{~h} / \mathrm{sem} . \text { ou } 288 \mathrm{~h} / \mathrm{ano}\end{array}$ & \\
\hline $\begin{array}{l}\text { Outras atividades } \\
\text { coletivas }\end{array}$ & $\begin{array}{l}>/=3,75 \% \\
2 \mathrm{~h} / \mathrm{sem} . \text { ou } 108 \mathrm{~h} / \mathrm{ano}\end{array}$ & & \\
\hline Teóricas & $\begin{array}{l}>/=10 \% \\
6 \mathrm{~h} / \mathrm{sem} . \text { ou } 288 \mathrm{~h} / \mathrm{ano}\end{array}$ & $\begin{array}{l}</=20 \% \\
12 \mathrm{~h} / \mathrm{sem} \text {. ou } 576 \mathrm{~h} / \mathrm{ano}\end{array}$ & \\
\hline $\begin{array}{l}\text { Nos níveis Secundário } \\
\text { e Terciário }\end{array}$ & $\begin{array}{l}>/=10 \% \\
6 \mathrm{~h} / \mathrm{sem} . \text { ou } 288 \mathrm{~h} / \mathrm{ano}\end{array}$ & $\begin{array}{l}</=20 \% \\
12 \mathrm{~h} / \mathrm{sem} . \text { ou } 576 \mathrm{~h} / \mathrm{ano}\end{array}$ & \\
\hline$\Sigma$ dos $\%$ mínimos & $75,5 \%$ & & \\
\hline$\Sigma$ dos $\%$ máximos & & $115 \%$ & \\
\hline
\end{tabular}


- Consultório de MFC: corresponde aos espaços institucionais reservados para a realização de consulta individual ou familiar no âmbito da APS e da MFC.

- Atenção domiciliar: corresponde às consultas e internações domiciliares.

- Grupos terapêuticos: correspondem às atividades de organização e realização de trabalho com grupos de pacientes/pessoas/famílias/comunidade, que contemplem a participação do residente e que tenham por objetivo: a informação; educação em saúde; incremento da autonomia; suporte terapêutico, relacionados a temáticas específicas e/ou grupos homogêneos.

- Administrativo-gerenciais: correspondem às atividades de organização dos prontuários médicos e outros registros como banco de dados das famílias e comunidade que propiciem a organização e o planejamento das ações de saúde; reuniões da equipe envolvida com o cuidado em saúde, bem como a gestão de serviços e sistemas locais de saúde.

- Outras atividades coletivas: correspondem às visitas domiciliares não-clínicas, cadastramento de famílias; contato com associações de moradores, participação em reuniões comunitárias, dos Conselhos de Saúde e outras instâncias de representação social da comunidade; ações de intersetorialidade.

- Teóricas: correspondem ao desenvolvimento de estudos dirigidos; cursos, discussões de casos e temas clínicos; seminários de integração teórico-prática; produção científica, trabalho de final de curso; jornadas, seminários, e congressos e atividades afins na área de interesse da MFC.

Nos níveis secundário e terciário da atenção: incluem o acompanhamento de pacientes sob regime de internação hospitalar e o atendimento em situação de emergência no âmbito da prática médica. A carga horária destinada a tais atividades, como o treinamento hospitalar, pode ser concentrada em períodos mensais, semestrais ou anuais durante o desenvolvimento da residência.

Competências a serem constituídas durante o PRMFC, cenários de treinamento e atividades teórico/práticas.

Os quadros a seguir apresentam os objetivos gerais, os conhecimentos, as habilidades, atitudes, bem como os requisitos mínimos para o treinamento em serviço e desen- volvimento das atividades teórico-práticas recomendados pela SBMFC no tocante a dispensação de cuidados, seja no âmbito individual ou coletivo. Para efeitos didáticos, estes quadros foram sistematizados em áreas, a saber:

- Competências Clínicas na Atenção Primária nas áreas da Saúde da Mulher; do Homem; do Adulto; do Idoso; da Criança; do Adolescente.

- Competências Clínicas da Atenção Secundária.

- Competências Clínicas na Atenção Terciária e Emergência.

- Abordagem Sistêmica da Família.

- Abordagem Comunitária.

A abordagem de cada área deverá ser desenvolvida com base nas melhores evidências disponíveis, fundamentando processos decisórios, particularizados e contextualizados por meio do diálogo permanente com os princípios e as estratégias da Medicina baseada em narrativa.

Cenários de prática e infra-estrutura necessária ao desenvolvimento de programas de residência em Medicina de Família e Comunidade

Para o desenvolvimento de um programa de residência médica, é indispensável dispor de uma estrutura compatível com o desenrolar de um processo ensino-aprendizagem de qualidade, tanto no tocante ao treinamento em serviço quanto ao desenvolvimento das atividades teóricas. As variações quanto ao espaço físico e demais recursos diferem de um programa de residência para outro, em função da especificidade de cada especialidade médica e de cada local.

Em relação à residência de Medicina de Família e Comunidade, a SBMFC recomenda a seguinte estrutura:

a) Unidade de Saúde para treinamento em serviço no âmbito da Clínica da MFC.

Locais ou espaços onde serão desenvolvidas atividades no nível da Atenção Primária à Saúde, especialmente consultórios destinados à prática da consulta médica. Tais espaços podem ser exclusivos para tal finalidade. Neste caso configuram Unidades de Medicina/Saúde da Família, também denominadas de Unidades Básicas de Saúde. Mas também podem estar situados próximos ou na mesma edificação de outras unidades de cuidados primários, secundários ou 


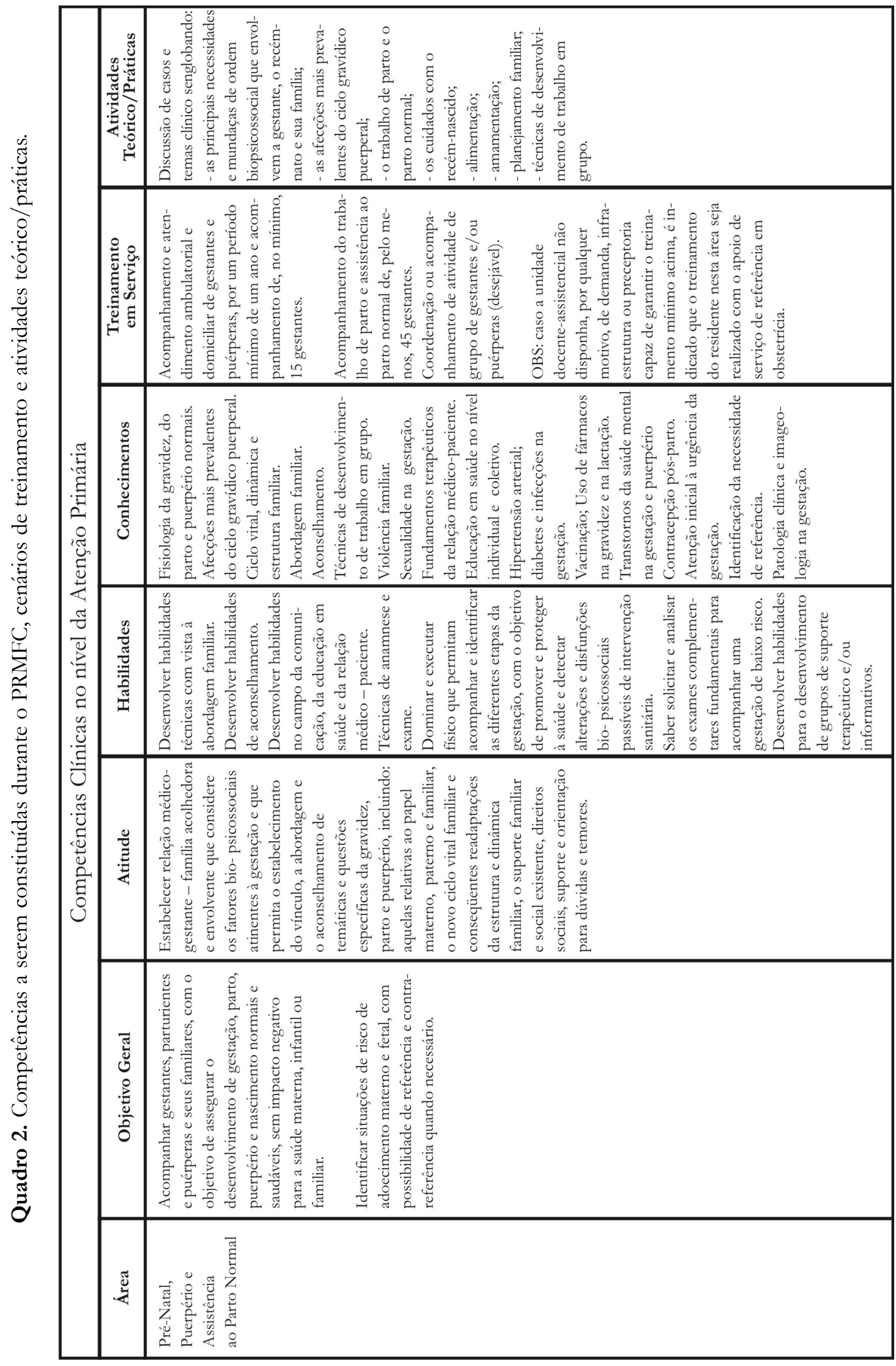




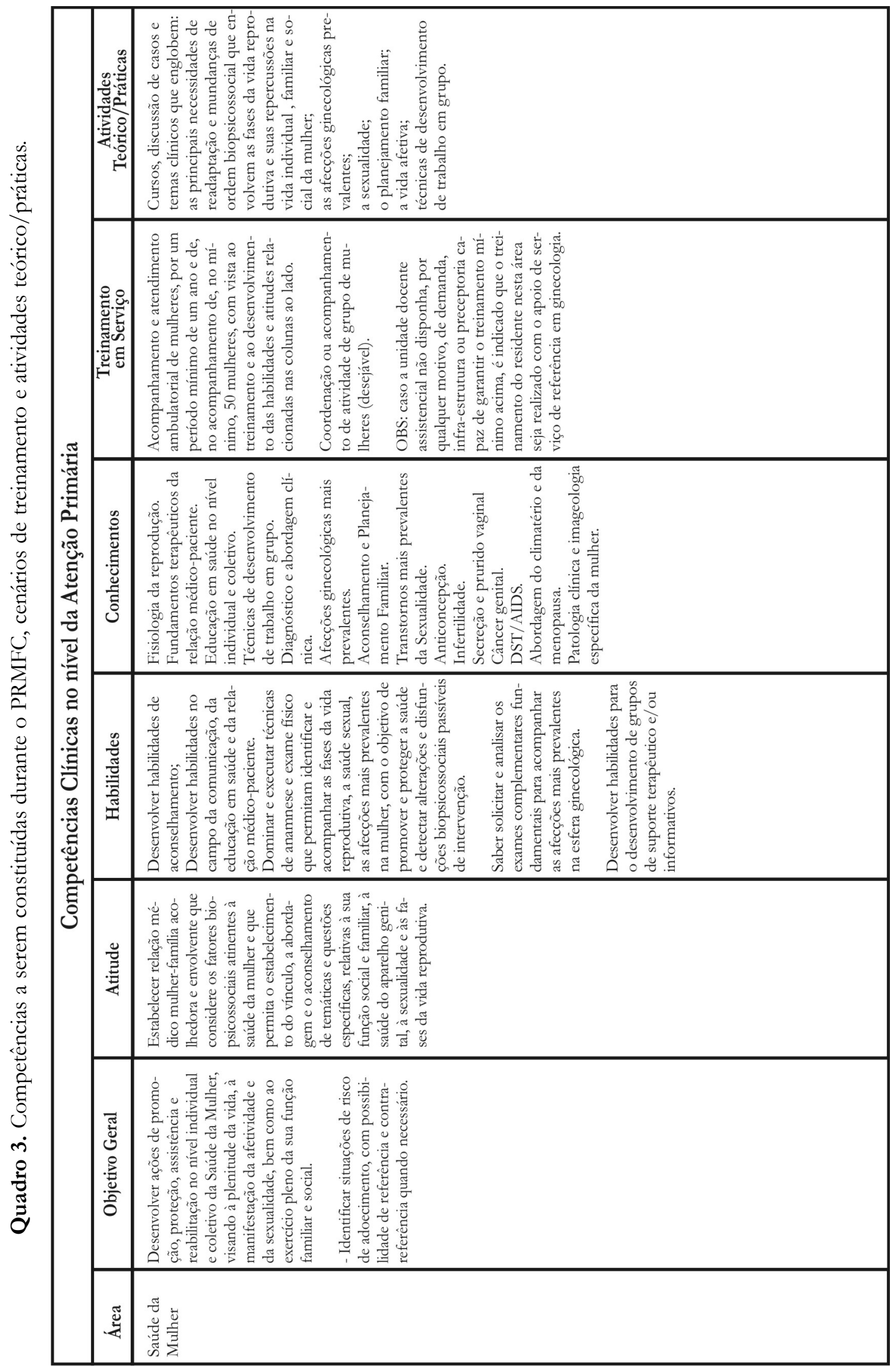




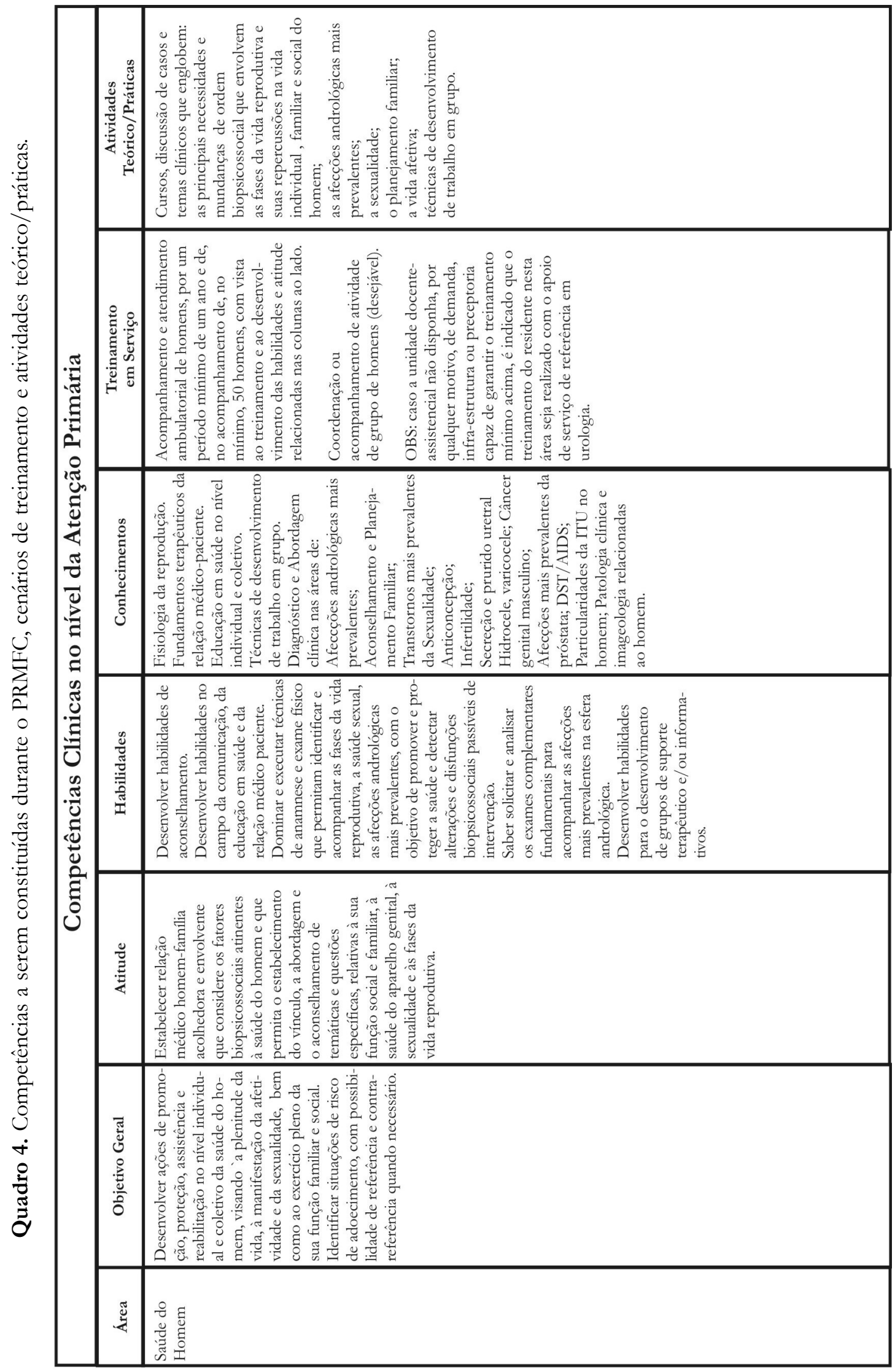




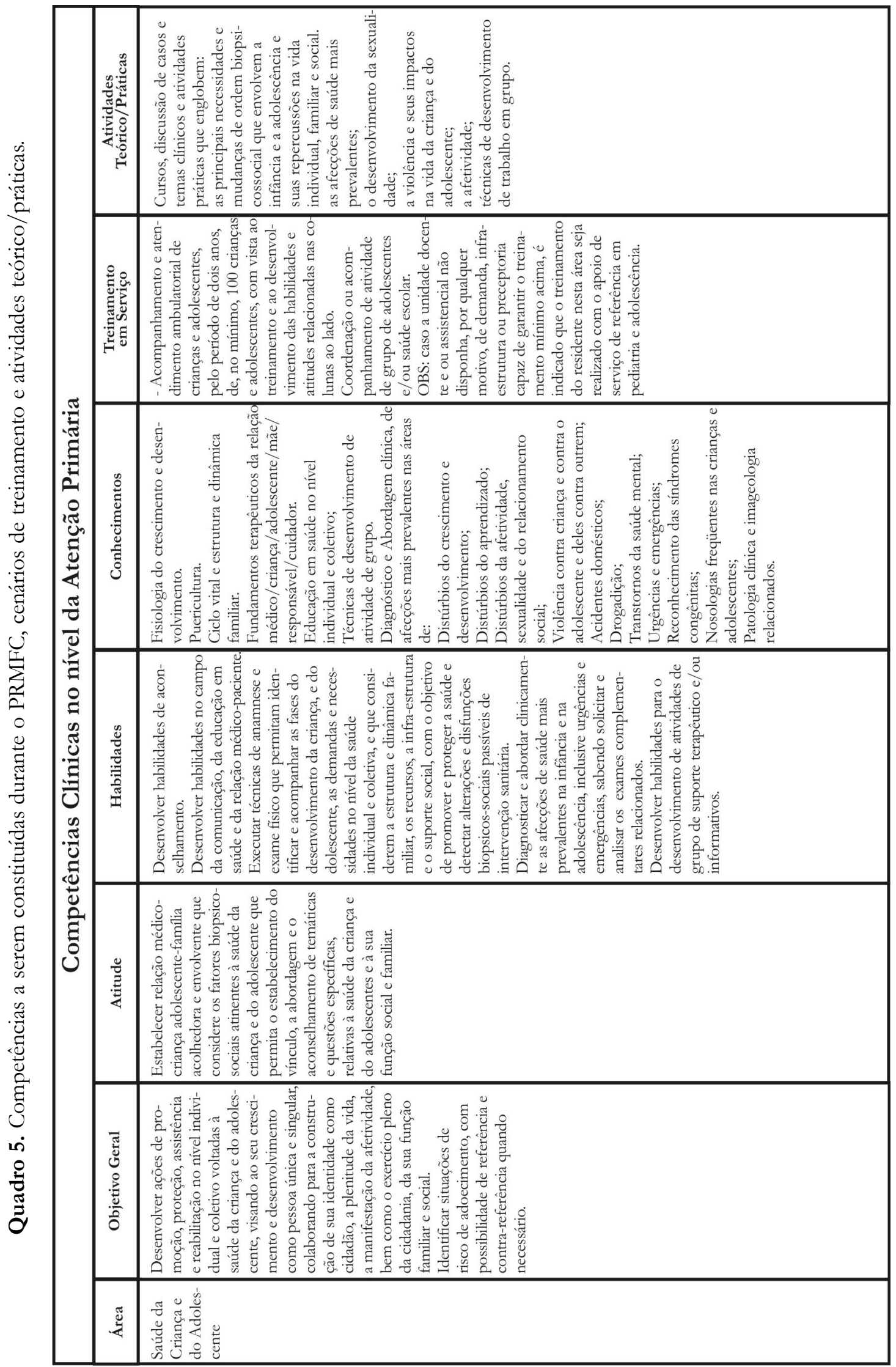




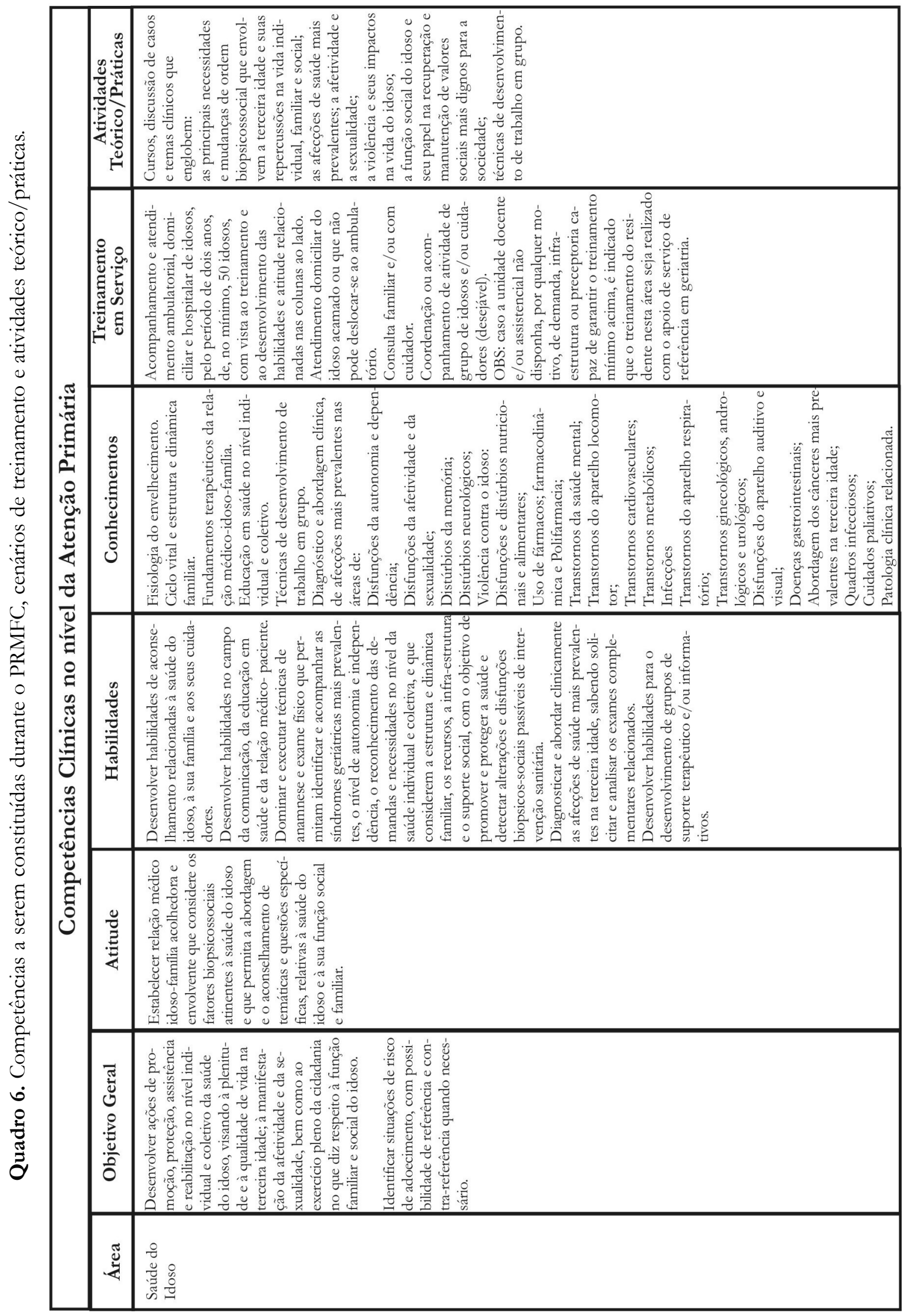




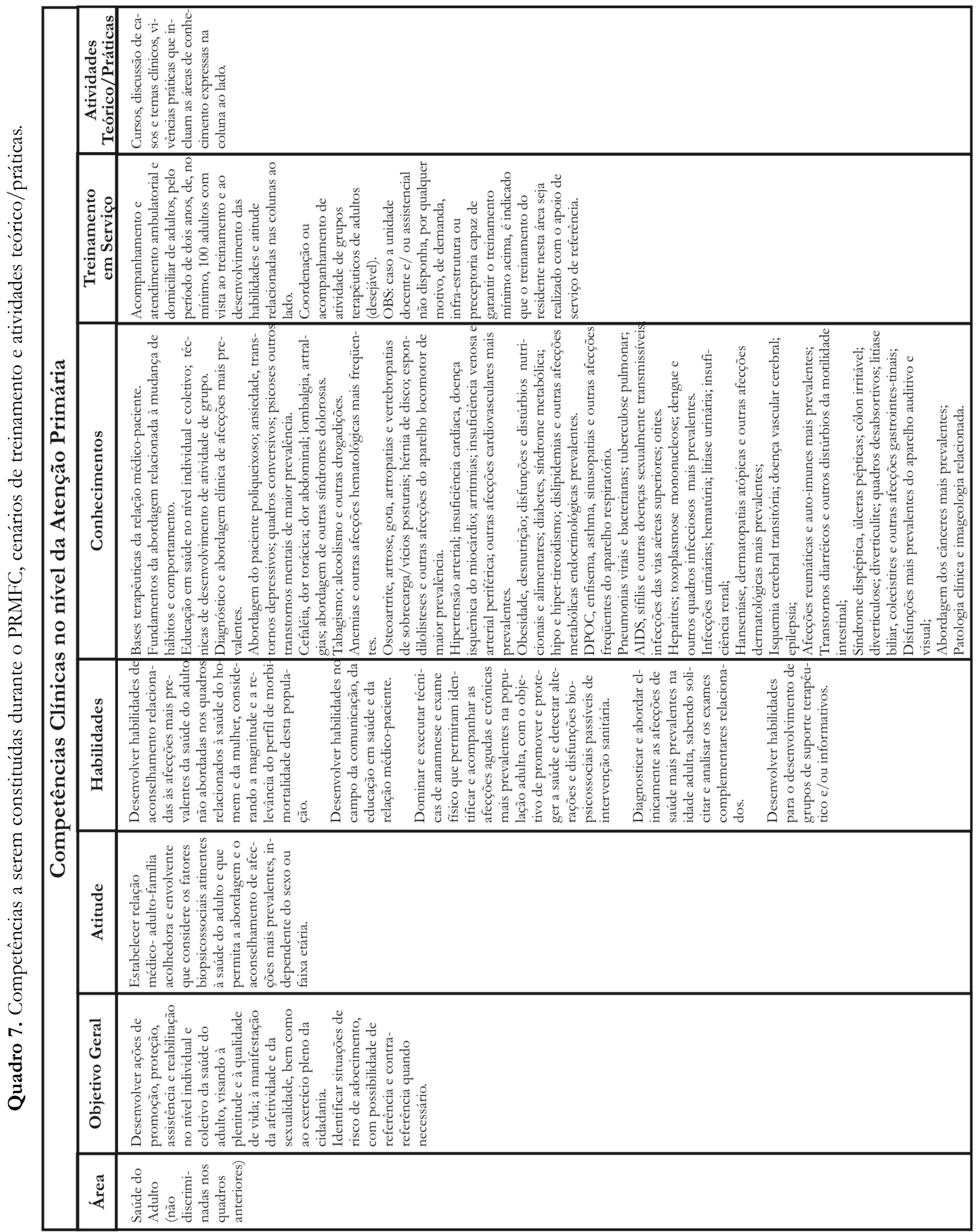

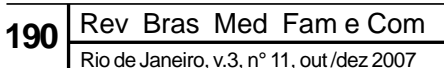




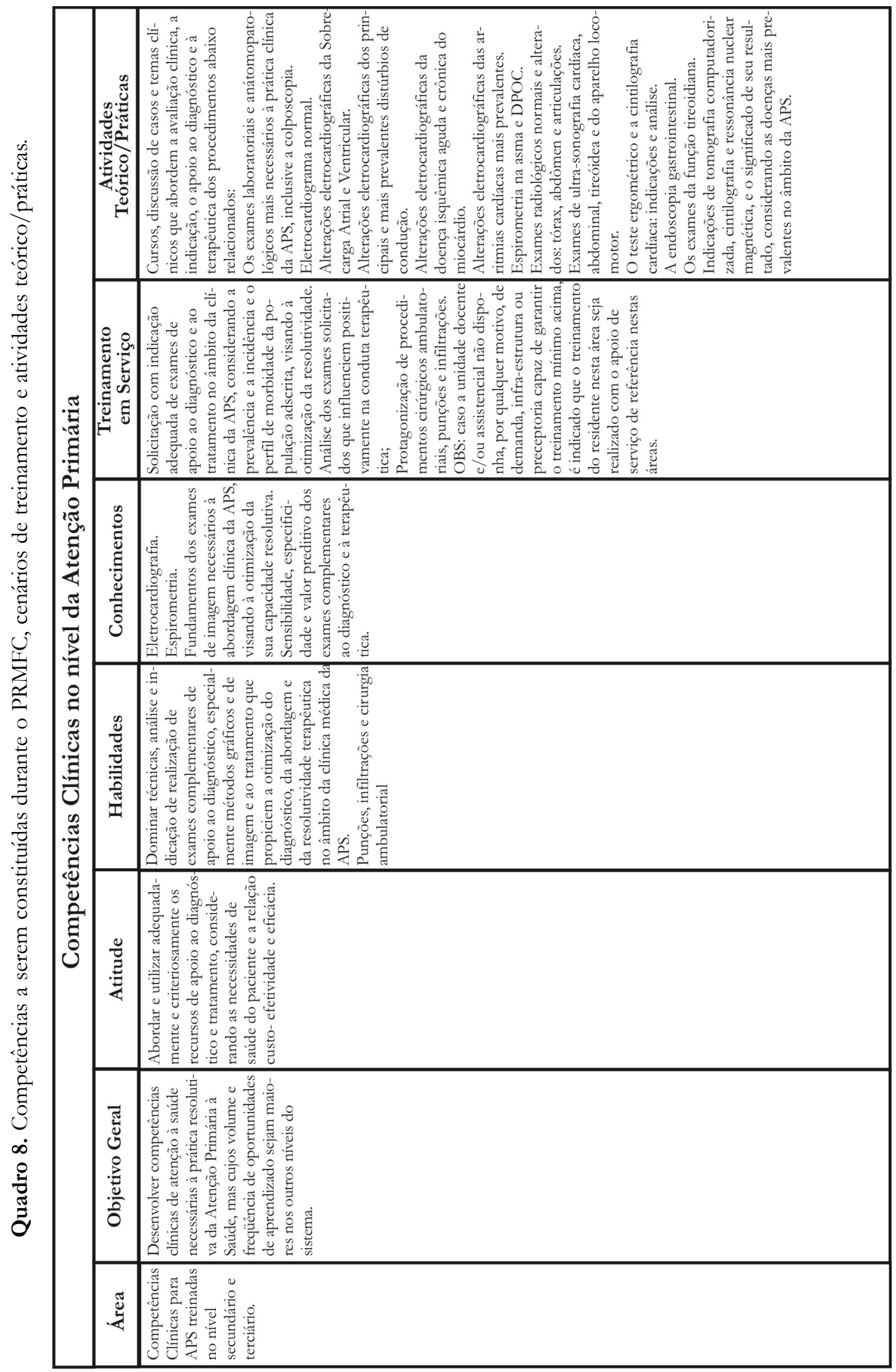




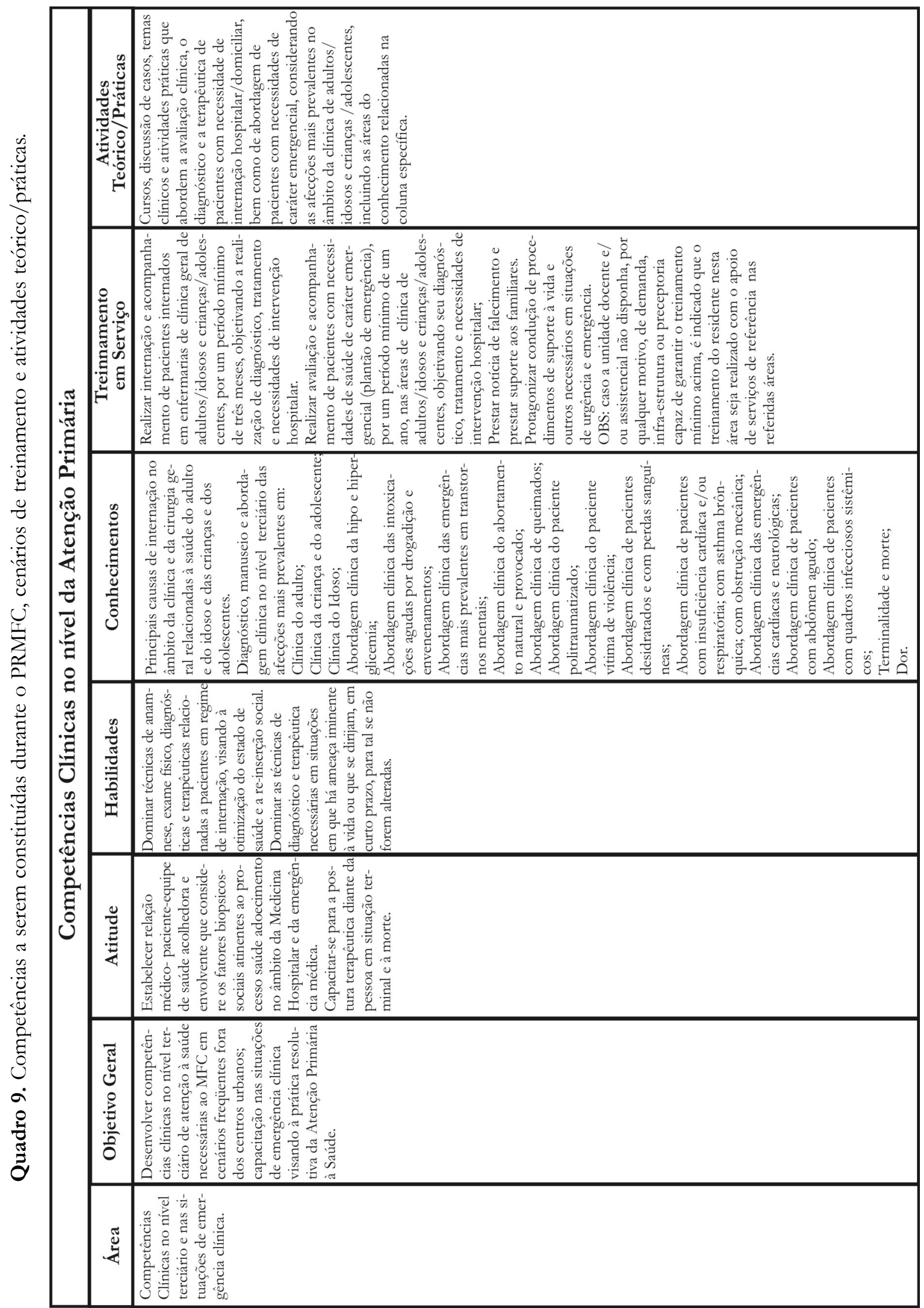




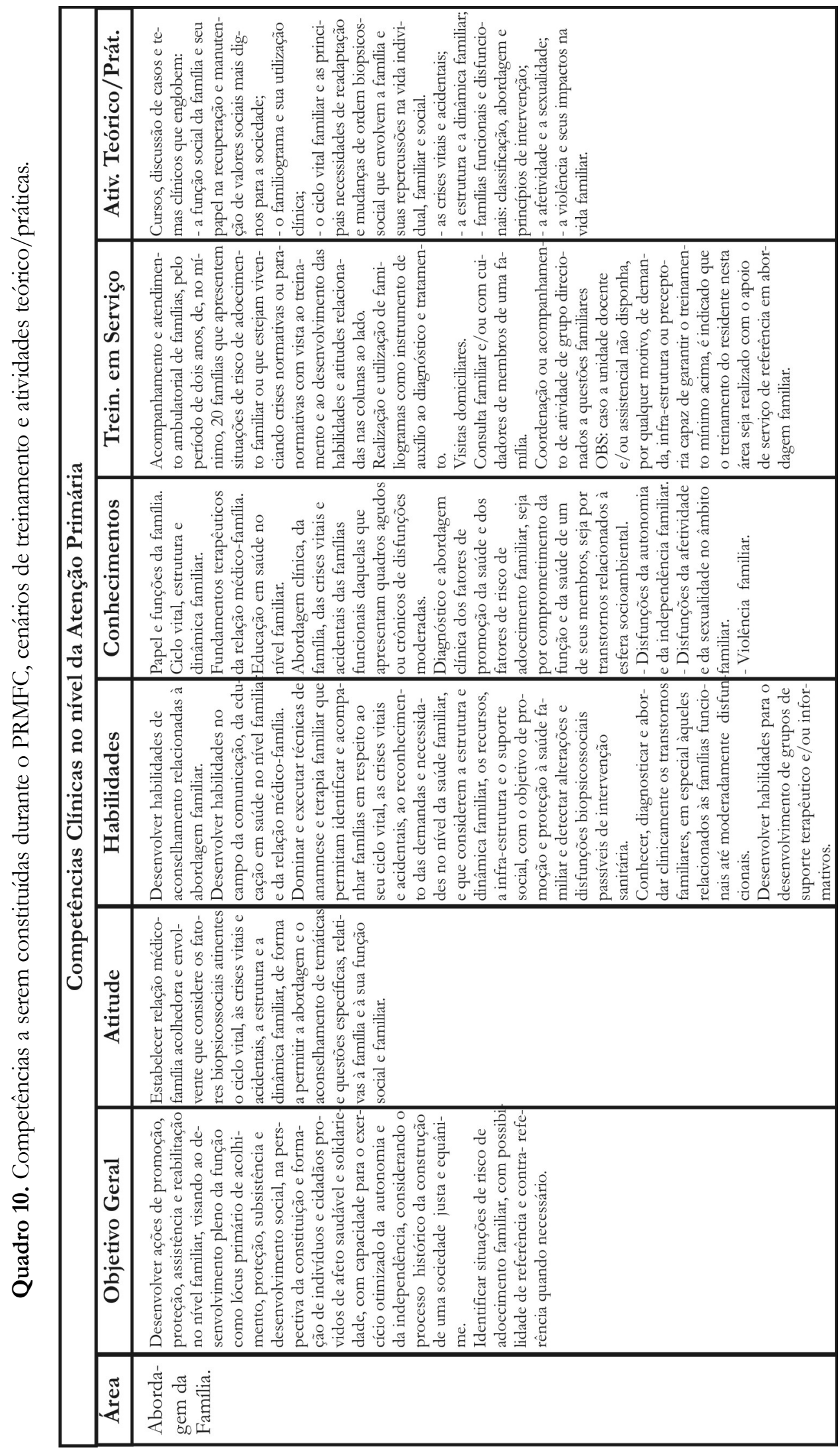




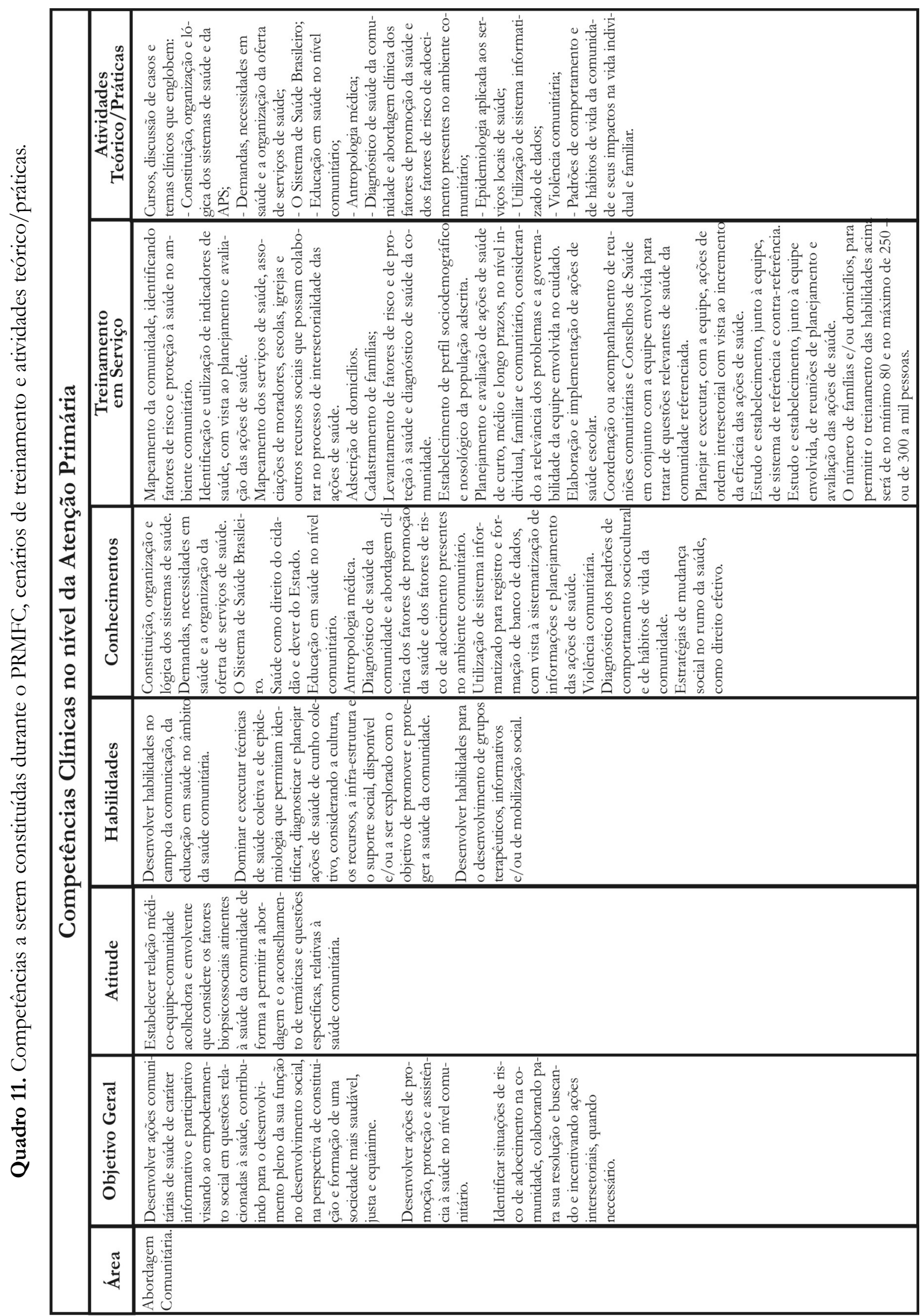

194 \begin{tabular}{l|l}
\hline \multirow{2}{*}{ Rev Bras Med Fam e Com } \\
\cline { 2 - 2 } & Rio de Janeiro, v.3, n 11, out/dez 2007
\end{tabular} 
terciários de saúde, a exemplo de postos ou centros de saúde, policlínicas e unidades ambulatoriais de hospitais.

Devem ser adequados e bem preparados para o atendimento de crianças, adolescentes, adultos e idosos. O cenário adequado seria constituído por:

\section{Planta física e instalações}

- Sala de espera.

- Recepção.

- Espaço para arquivamento das fichas, prontuários e outros registros de organização e planejamento do trabalho. - Consultórios (mínimo de $7,5 \mathrm{~m}^{2} /$ consultório) para atendimento infantil, gineco-obstétrico e clínica de adultos.

- Sanitários.

- Sala para desenvolvimento de atividades de grupo e atividades teóricas (que comporte de 12 a 20 pessoas e tenha de 9 a $20 \mathrm{~m}^{2}$ ).

- Ventiladores e ar-condicionado.

- Instalações elétricas e iluminação adequados.

\section{Mobiliário, equipamentos e outros recursos}

- Mesas, cadeiras, mesa ginecológica, escada de apoio e foco de luz (consultório gineco-obstétrico), balança de adulto, mesa de exame clínico pediátrico e balança para recém-natos (consultório pediátrico), biombo, mesa de exame clínico e escada de apoio; estante(s); arquivos mesa para computador.

- Computador e impressora, telefone; conexão para internet.

- Esfingmomanômetro, estetoscópio, termômetro, fita métrica, sonar, estetoscópio de pinard, otoscópio, oftalmoscópio, laringoscópio, maleta, sonar doppler obstétrico, microscópio (desejável).

- Tubo orotraqueal, ambu, microscópio, bandeja para pequenos procedimentos em cirurgia, bandeja para cateterismo vesical, material para exame ginecológico (espéculos, pinça longa, lâmina, espátula, escova), lanterna, foco pescoço de ganso, oxigênio, nebulizador, aspirador, oxímetro de pulso, aparelho para eletrocardiografia (desejável), manguitos para esfingmomanômetro conforme faixa etária e para paciente obeso, ventilador/aquecedor, cortinas.
6. Disponibilidade mínima de livros para consulta clínica

- Duncan, Schmidt, Giuliani Medicina Ambulatorial Ed. Artes Médicas, 3a edição, 2004.

- Soares, J.L.F.; Pasqualotto, A.C.; Leite, V.R.S. Métodos diagnósticos - Consulta rápida. Porto Alegre: ArtMed Editora, 2002.

- PROMEF - Editora Artmed.

b) Unidades de referência/contra-referência

$\mathrm{Na}$ sua formação, o médico de Família e Comunidade deverá ser capaz de cumprir o programa mínimo estruturado para a especialidade, incluindo a preceptoria e as competências no nível dos cuidados secundários e terciários de saúde, e, também, dispor de referência e contra-referência de pacientes quando necessário.

\section{Os principais serviços para atender a essas neces-} sidades incluem:

- Pediatria.

- Ginecologia / Obstetrícia.

- Medicina interna.

- Pequenas cirurgias.

- Saúde mental.

- Unidades de emergência.

- Unidades hospitalares (pediatria, clínica médica, cirurgia geral e ginecologia/obstetrícia).

c) Acesso a métodos de investigação diagnóstica

No desenvolvimento de sua prática, o médico de Família e Comunidade necessita desenvolver senso crítico, conhecer e aplicar métodos de investigação diagnóstica, não só para sua formação, mas também para atingir o grau de resolutividade inerente à prática de excelência da especialidade, que corresponde a, no mínimo, $85 \%$ dos problemas de saúde de uma determinada população.

Para tanto, o acesso aos meios diagnósticos é condição essencial. Dentre os principais, estão:

- Métodos de imagem: radiologia, ultra-sonografia; cintilografia.

- Endoscopia digestiva e intestinal. 
- Métodos gráficos: eletrocardiografia e ergometria de esforço.

- Patologia clínica:

Hemograma completo, pesquisa de leucócitos atípicos, monoteste, contagem de reticulócitos, ferro sérico, transferrina, ferritina, TIBIC, velocidade de hemossedimentação, lipidograma, coagulograma, dosagem/titulação de sódio, potássio, cálcio, ácido úrico, fator reumatóide, FAN, Proteína C Reativa, ASLO, uréia, creatinina, glicemia, hemoglobina glicosilada, hormônio tireoestimulante, T4 livre, amilase, CK, CKMB, transaminase oxalacética, transaminase pirúvica, fosfatase alcalina, proteínas totais e frações, tipagem sangüínea, fator Rh, sorologias (HIV, hepatite $\mathrm{B}$, hepatite $\mathrm{C}, \mathrm{EBV}, \mathrm{CMV}$, toxoplasmose, doença de Chagas), VDRL, FTA-ABS, teste de coombs, clearance de creatinina, proteinúria, EAS, urinocultura, TSA, PSA total, parasitológico de fezes, sangue oculto nas fezes, TSA.

d) Atividades teóricas - Discussão de casos, cursos, oficinas e outros

Os programas de residência médica devem dedicar de $10 \%$ a $20 \%$ do seu tempo ao desenvolvimento de atividades de cunho teórico, com o objetivo de aprofundar conhecimentos mais específicos da especialidade, considerando sua aplicabilidade prática. Neste campo, as ferramentas da MFC devem ser objeto destes espaços. Necessariamente, devem ser incluídos:

- A pessoa, os ciclos de vida e a abordagem integral à saúde.

- Família e instrumentos de abordagem familiar.

- Comunidade e instrumentos de abordagem comunitária.

- Clínica do MFC.

- Educação em saúde.

- Gerência e avaliação de serviços locais de saúde.

Nas tabelas descritivas das competências do MFC, apresentadas anteriormente, a última coluna (Atividades Teóricos/Práticas) pode servir de orientação sobre as temáticas mais relevantes para o desenvolvimento dessas atividades. Vale destacar que os melhores resultados deste tipo de atividade estão na dependência, também, do pro- cesso pedagógico a ser utilizado. Deve ser considerado, neste caso, a forma de aprendizado dos adultos (residentes), o qual envolve o aprendizado significativo, com base em problemas concretos e reais enfrentados no dia-a-dia. Desta forma, a Discussão de Casos - de pessoas ou famílias atendidas, situações vivenciadas nos domićlios, na comunidade, seguidos e/ou precedidos de leitura e/ou sistematização de textos informativos - pode se configurar uma boa opção para estruturar estas atividades.

e) Infra-estrutura docente

A função de preceptoria requer, igualmente, a disponibilidade de estrutura adequada para seu desenvolvimento.

Considerando este ponto de vista, são recursos necessários:

- Acesso à comunicação imediata (linha telefônica).

- Material educativo (quadro branco; pilots; papel; canetas; retroprojetor; recursos de mídia).

- Acesso à internet.

- Biblioteca.

\section{Atividades de preceptoria, normas transitórias e capacitação de preceptores}

Todo processo de expansão acelerada supõe, antes de tudo, uma necessidade que o justifique. Essa necessidade foi delineada na introdução deste projeto. Diante da defasagem de preceptores especializados em MFC e a crescente demanda de abertura de programas, é recomendável fazer uma adaptação transitória da norma vigente (CNRM), visando a preservar seu objetivo sem bloquear o atendimento da necessidade social de formação desse especialista em escala ampliada.

A SBMFC entende que é necessário que o conjunto dos médicos que vierem a assumir a supervisão direta de residentes em novos programas inicie formação como preceptores antes do início dos mesmos. E também que, até o final do credenciamento provisório, titulem-se como especialistas, recebendo o apoio necessário para essa conquista. Já os coordenadores teriam até o primeiro recreden- 
ciamento dos respectivos programas para obterem a mesma titulação. Estas recomendações assumem ainda maior relevância à medida que se entende que a preceptoria é uma atividade eminentemente presencial, sobretudo, no que toca às práticas de consultório, considerando-se em caráter de exceção algumas situações específicas. Mesmo nesses casos que fogem à regra não se pode dispensar a preceptoria presencial em grande parte das atividades desenvolvidas pelo residente.

A SBMFC entende que é necessário e pertinente o incentivo a outras formas de incrementar o processo ensino-aprendizagem, inclusive pelo acesso do residente ao preceptor via meios rápidos de comunicação, como telefone e/ou internet. Além disso, parece aceitável em algumas circunstâncias, que um mesmo especialista compartilhe a preceptoria de residentes em unidades muito próximas, mas não se pode aceitar transgressão a norma sobre proporção entre o número de residentes e preceptores.

Embora a formação dos preceptores da área não possa prescindir do protagonismo da Sociedade da Especialidade, a SBMFC entende que há um outro campo de apoio à expansão simultânea da ESF e dos PRMs em MFC que deve ser compartilhado presencialmente e à distância por especialistas focais de várias áreas, assim como por MFCs experientes via recursos de telemedicina. Esta nova modalidade de supervisão será importante tanto para os médicos residentes como para os preceptores em formação. Nesta perspectiva, pediatras, internistas, tocoginecologistas, cirurgiões e psiquiatras ligados a hospitais e/ou centros de saúde de um município ou região podem ser mobilizados para supervisionar, presencialmente e/ou à distância, o atendimento de casos menos freqüentes, ou mais complicados, realizado pelos residentes nas Unidades de Saúde da Famíliain ${ }^{12,13}$.

A disponibilidade de computadores e comunicação pela internet cria condições apropriadas para a discussão de casos e avaliações conjuntas de situações por colegas mais experientes ou com maior formação em determinadas áreas. Para isso, é essencial adotar estratégias de qualificação que possibilitem a serviços de Atenção Primá- ria com reconhecido padrão de excelência candidatar-se para prestar esta modalidade emergente de apoio à distância, obviamente, recebendo recursos públicos necessários para tal.

A tudo isso deve ser acrescentada a necessidade de assegurar apoio e facilitar o acesso de preceptores dos PRMs, em especial àqueles localizados em áreas menos providas de transporte, a atividades educacionais reconhecidas no processo de revalidação de seu título de especialista. Aqui se incluem tanto as atividades presenciais como aquelas à distância.

Com o objetivo de colaborar com o processo de capacitação de preceptores e de qualificação da prática da preceptoria em RMFC e outros cursos de especialização na área, a SBMFC estruturou uma Oficina de Multiplica- dores, ainda em 2005, e a seguir iniciou um processo de Capacitação de Preceptores. Estas Oficinas de Capacitação vêm sendo coordenadas por Carmem Fernandes, José Mauro Cerati Lopes, Leda Chaves Dias Curra e Luiz Felipe C Mattos, especialistas em MFC do Rio Grande do Sul, que organizaram um detalhado manual de apoio pedagógico para este fim $^{14}$.

O público-alvo da Oficina de Multiplicadores foi composto por médicos que tinham cursado residência médica na especialidade e que, preferencialmente, eram preceptores de programas de RMFC já existentes.

O público-alvo do Curso de Capacitação de Preceptores, por sua vez, é constituído por médicos especialistas em MFC, ou outros que estejam envolvidos ou venham a se envolver com a preceptoria ou a docência clínica da Atenção Primária, Saúde e Medicina de Família e Comunidade, em especial ex-residentes ou residentes de segundo ou terceiro ano de PRMFC.

Vale acrescentar, por fim, que este processo de capacitação, objeto de outro documento da SBMFC, resultou, até o final de 2007, em 30 multiplicadores e cerca de 200 preceptores capacitados, números que apontam para a excelente aceitação da estratégia. 
Maria Inez Padula Anderson, Eno Dias de Castro Filho, Ricardo Donato Rodrigues, Marcello Dala Bernardina Dalla e Monique Marie Marthe Bourget

\section{Referências}

1. Falk JW. A Medicina de Família e Comunidade e sua Entidade Nacional. histórico e perspectivas. Rev Bras Med Família e Comunidade. 2004; 1 (1).

2.Penaforte J, Bessa O. Apresentação in Médico de Família. Formação, Certificação e Educação Continuada. Fortaleza (CE): Escola de Saúde Pública do Ceará; 2002. p.1116.

3.Anderson MIP. A Atenção Primária à Saúde que Queremos. 2005. $7^{\circ}$ Congresso Brasileiro de Medicina de Família e Comunidade. Belo Horizonte (MG), SBMFC; 2005. 4.Anderson MIP. Primary Care and Family and Community Medicine in Brazil. Florence: WONCA EUROPE; 2006. 5.Brasil. Medida Provisória $\mathrm{N}^{\circ} 411$ de 28 de dezembro de 2007. Brasília: DOU de 28/12/2007 - Edição extra. Disponível em: http://www010.dataprev.gov.br/sislex/ paginas/42/2005/11129.htm

6.Brasil. MS/MEC Portaria Interministerial $\mathrm{N}^{\circ}$ 1597, de 22 de julho de 2007. Disponível em: http:// dtr2001.saude.gov.br/sas/PORTARIAS/Port2007/GM/ GM-1507.htm

7.Brasil. Portaria n 649/MS, de 28 de março de 2006. Disponível em: http://dtr2004.saude.gov.br/dab/docs/ legislacao/portaria_649_28_03_2006.pdf

8.Brasil. Ministério da Saúde. Portaria 1.111/GM, de 5 de junho de 2005. Disponível em: http:/ /dtr2001.saude.gov.br/ sas/portarias/Port2005/GM/GM-1111.htm

9.Hadadd AE. Residência em Medicina de Família e Comunidade. 2006. $8^{\circ}$ Congresso Brasileiro de Medicina de Família e Comunidade. São Paulo, SBMFC; 2006.

10.Anderson MIP, Demarzo M, Rodrigues RD. A Medicina de Família e Comunidade, A Atenção Primária à Saúde e o Ensino de Graduação: Recomendações e Potencialidades. Rio de Janeiro: SBMFC; 2005. 32p. Disponível em: www.sbmfc.org.br

11.Brasil. Ministério da Educação. Resolução 04/2003/ CNRM. Disponível em: http://portal.mec.gov.br/sesu/ arquivos/pdf/cnrm_042003.pdf

12.Brasil. Programa Nacional de Telessaúde. Disponível em: http://www.telessaudebrasil.org.br/php/index.php
Bases para expansão e desenvolvimento adequados de programas de Residência em Medicina de Família e Comunidade
13. Anderson MIP, Monteiro A, Joao Junior M, Gismondi R. O Estado do Rio de Janeiro, A Faculdade de Ciências Médicas da Universidade do Estado do Rio de Janeiro e a Estratégia de Saúde da Família. Experiências em Telemedicina e Telessaúde. In: Alaneir FS, Souza C, Alves HJ, Santos FS. (Org.). Telessaúde. Um Instrumento de suporte Assistencial e Educação Permanente. Minas Gerais: Editora MG; 2006. p. 290-295.

14. Sociedade Brasileira de Medicina de Família e Comunidade. Portal. Disponível em: http://www.sbmfc.org.br

\section{Endereço para Correspondência:}

Rua 28 de setembro, sala 605,

Rio de Janeiro RJ

CEP: 20.551-031

\section{Endereço Eletrônico:}

inezpadula@terra.com.br 